\title{
Microsatellite analysis to evaluate the seedling and mature of oil palm populations (Elaeis guineensis Jacq.) to Ganoderma boninense resistance
}

\author{
Rahmah Hayati ${ }^{1}$, Lisnawita Lisnawita ${ }^{2}$, Erman Munir ${ }^{3}$, Mohammad Basyuni ${ }^{4, *}$ \\ ${ }^{\prime}$ Doctoral Program of Agricultural Sciences, Faculty of Agriculture, Universitas Sumatera \\ Utara, Medan, Indonesia \\ 2 Program Study of Agrotechnology, Faculty of Agriculture, Universitas Sumatera Utara, \\ Medan, Indonesia \\ ${ }^{3}$ Dept. of Biology, Faculty of Mathematics and Natural Sciences, Universitas Sumatera Utara, \\ Medan, Indonesia \\ ${ }^{4}$ Dept. of Forestry, Faculty of Forestry, Universitas Sumatera Utara, Medan, Indonesia \\ *Corresponding author: m.basyuni@usu.ac.id
}

\begin{abstract}
To conduct efficient disease screening, the differentiation of the palm tree (Elaeis guineensis Jacq.) to Ganoderma boninense resistance populations is critical. Differentiation can be done by analyzing the genetic structures of the two populations and comparing their seedling and matures. This article presents the results of studying four selected stably amplified polymorphic of codominant microsatellite data to estimate the differentiation between eight E. guineensis populations from different types of inoculated or symptoms of Ganoderma from North Sumatera, Indonesia. The DNA extraction was carried out based on the CTAB method with a little modification. The forward and reverse primers, i.e., P4(EGIFR), P6(EG01), P7(EG02), and P9(EG03), were used in a polymerase chain reaction (PCR). The detection of the results of amplification was performed using molecular weight analysis on UVITEC-1D software. To determine the microsatellite analysis, the data of genes were used to perform with the GENALEX ver 6.502 and MVSP ver 3.2 software. Four of the loci have polymorphism for all types of seedling and mature species of $E$. guineensis. The polymorphism information content was calculated as $\mathrm{PIC}=0.70$. The genetic differentiation among populations was highest (Fst $>0.25$ ), showed a high genetic divergence. The calculation of Fst by population revealed that the highest Fst was largely due to suggesting diversifying selection in gene flow and resistance selection. The molecular of analysis variance (AMOVA) was showed a significant (5\%) differentiation among individuals $(100 \%)$ between eight types of population. A clustering by UPGMA analysis, based on squared Euclidean and Datalog (10), transformed genetic distances and revealed was a significant difference cluster in the seedling and mature populations.
\end{abstract}

Keywords: Codominant data; Elaeis guineensis; ganoderma; genetic diversity; seedling and mature.

\section{Introduction}

The oil palm plantation is a highly important commodity in tropical countries for generating foreign exchange (Sayer et al., 2012; Phalan et al., 2013). Elaeis guineensis Jacq. is an eternal, fat-producing species of African origin, amounting to $30 \%$ of Africa's vegetable oil output, and has been increased demand in 2019 (Voora et al., 2020). The oil palm can be live more than 20 years to yields production for the crude palm oil (CPO), to increase the agro-industries (Saktioto et al., 2019). The global production of oil palm in 2019 was 75.7 million tons in Indonesia, wherein the oil palm production was approximately 42.7 
million tonnes and generated about 21-23 $(\mathrm{w} / \mathrm{w})$ in each ton of fresh fruit bunches (FFB) (Indonesian Directorate General of Estate 2020; Shahbandeh, 2020). However, basal stem rot fungus, Ganoderma boninense, has been now revealed to be a major threat to E. guineensis plantations in North Sumatra, Indonesia (Hayati et al., 2020). Oil palm plantations considerable losses caused by the impact of disease of $G$. boninense (Caro et al., 2014), the use of resistant planting materials is needed (Purba et al., 2019).

For a long time, the Ganoderma disease was tackled using planting material resistant to disease (Mudge et al., 2020). Several researchers still examined the quality and performance of the plant breeders of $E$. guineensis to finding the germplasm resources of Ganoderma resistance. Oil palm cultivation is one of the most profitable and the molecular bases of important agronomic traits at the single base-pair resolution, enabling gene-centered breeding and engineering this remarkable crop (Barcelos et al., 2015). Certain marker types may be used to perform genetic screening approximation, and the molecular marker as single nucleotide polymorphism (SSR) has been widely applied to the study of genetic structure among and also within-population to diverse plant species (Maughan et al., 1995; Powell et al., 1996 and Sserumaga et al., 2019). The MAS (Marker Assisted Selection) targets the genes and utilizes the probe-level of genetic structure and diversity among a population of tightly related species available in a bank of germplasm (Mullis, 1990; Erlich et al., 1990).

The simple sequence repeats (SSR) marker was the higher polymorphic, specific of the genome, codominant data, and indicated a nearly instead distribution more to the genome. It is used in the genetic diversity analysis in structure population, assisted selection for crop improvement, and gene mapping (Madar et al., 2019; Osorio-Guarín et al., 2019; Terryana et al., 2020). Currently, SSR markers are one of the most promising molecular marker systems for understanding the genetic diversity and structure of oil palm (Singh et al., 2008). The study was provided a theoretical and experimental basis for future breeding, evaluation, management, and conservation of oil palm.

Faizah et al., 2020, has considered that resistance to basal stem root (BSR) would quickly be overwhelmed by the large volume of inoculum present in many plantations. However, the genetic improvement has provided resistance to the diseases prevalent in oil palm nursery stocks. Thus, it can be reduced using resistant materials. For Ganoderma diseases, a relatively rapid screening method was available for breeding programs (Teh et al., 2020). In addition, the observations on resistance to BSR thus far were depended on the incidence of natural infections in the field, a process needed for many years. The root inoculation method was provided as an alternative to screening. Recently, Afandi et al., 2018, reported the oil palm resistance gene seedling and mature variety based on the biochemistry characteristics. However, the genetic diversity of seedlings and mature populations infect with $G$. boninense is not fully understood. The present study was described the genetic diversity and structure of four population seedlings and matures of oil palm that $G$. boninense inoculation.

\section{Materials and methods}

\subsection{Root samples}

Eight types of oil palm root tissue samples with three replicated were selected from experimental genetic materials of Elaeis guineensis Jacq, located in the Socfindo $3^{\circ} 19^{\prime} 58^{\prime \prime}$ North Latitude, 99 02'25" East Longitude, North Sumatra, Indonesia. Four types of seedling and mature plants (15 years after planting) were selected.

Seedling resistance (SR), seedling non-resistance $(\mathrm{SN})$, seedling resistance inoculation Ganoderma (SRIG), and seedling non-resistance inoculation Ganoderma (SNIG) were planted and harvested for 112 days (Ganoderma boninense inoculated in 56 days after planting (DAP)). Furthermore, the mature resistance symptom (MRs), mature resistance non-symptom (MRN), mature 
non-resistance symptom (MNs), mature non-resistance non-symptom (MNN) were 15 planting years. The roots were briefly kept in the nitrogen until analysis.

\subsection{DNA extraction}

The tissues (500 $\mathrm{mg}$ of roots per sample) were crushed and digested in $1 \mathrm{~mL}$ of cetyl trimethyl ammonium bromide (CTAB) method with a little modification. The content was included to $200 \mathrm{mM}$ Tris- $\mathrm{HCl}$ pH 8,0 mM EDTA, 4.5\% PVPP, 2\% CTAB, $1 \mathrm{M} \mathrm{NaCl}$ (CTAB $2 \times$ buffer), according to Neuhauser et al., 2009. The DNA was precipitated using 100\% ethanol (Zeugin et $a l ., 1985)$, the pellet was washed with $70 \%$ ethanol, then air-dried and eluted with Tris$\mathrm{HCl} 10 \mathrm{mM}$ pH 8.0 and EDTA $1 \mathrm{mM}$ pH 8.2. Finally, it was kept at $-20^{\circ} \mathrm{C}$ before used.

\subsection{DNA analysis}

The DNA quality was measured with electrophoresis; $3 \mu \mathrm{L}$ DNA was separated with loading dye in a $1.0 \%$ agarose gel (Lee et al., 2012) and defiled with GelRed, Biotium, (Fremont, CA) and shown under UV light Translumination (Imaging Systems). The DNAs were quantified in absorbance ratios (A280/260) and measured with the NanoPhotometer ${ }^{\circledR}$ N60/N50 (Rana \& Jain, 2019).

\subsection{PCR amplification}

The primers were used to amplify 240-395 bp to $E$. guineensis Jacq. resistance of Ganoderma boninense. Primer P4(EGIFR); F (5'AAGCTCCTGGACGACTTCAA-3') and R (5'-ATGGGAGGAAGTAACCAGCA-3') (Tan et al., 2013). Primer P6(EG01), P7(EG02), and P9(EG03) based on the previous study. P6(EG01); F(5'-TTGCTGGAGGAATGCTCTCT-3') R(5'-TCCAGCAGATGCAACACTTC-3'), P7(EG02);F(5'-AGGCCTACTTGGGTTCCACT-3') R (5'-GCCCTTTCACATGATGTCCT-3'), and Primer P9(EG03);F(5'-TCTTCTTCCCCTTCCTCCTC-3)R (5'-GGCAGTGCATTGGATGTCTA-3).
PCR was established under the conditions for denaturation at $94^{\circ} \mathrm{C}$ for $4 \mathrm{~min}, 35$ cycles at $94^{\circ} \mathrm{C}$ for $30 \mathrm{sec}$, annealing at $55^{\circ} \mathrm{C}$ for 45 sec, extension at $72^{\circ} \mathrm{C}$ for $1 \mathrm{~min}$, and then with a contained final extension at $72^{\circ} \mathrm{C}$ for $10 \mathrm{~min}$. One reaction contained $2.5 \mu \mathrm{L}$ Tiangen, Biotech (with component $10 \mathrm{mM}$ Tris- $\mathrm{HCl}$ in $\mathrm{pH} \mathrm{8.2,} \mathrm{5 \%} \mathrm{Glycerol,} 10 \mathrm{mM}$ EDTA $0.02 \%$ Bromophenol Blue, $1.0 \mu \mathrm{L}$ $\mathrm{F}$ and $\mathrm{R}$ primer, and $2.5 \mu \mathrm{L}$ DNA, $13.0 \mu \mathrm{L}$ $\mathrm{ddH} 2 \mathrm{O}$, for a final the volume of $20 \mu \mathrm{L}$.

\subsection{Electrophoresis}

PCR products were separated by electrophoresis in 1\% agarose gel (Lee et al., 2012). The allele was obtained in Gel-Red color, and a UV-Translumination carried out the image to assess the product molecular weight with UVITEC-1D software (Cambridge).

\subsection{Microsatellite data analysis}

The parameters of the genetic structures were calculated using GENALEX ver 6.502. This software was particularly in the range matrix to generate multiple types of the marker, including codominant data, haploid data, and binary genetic data. The SSR was used in codominant data sets and options and was used for all analyses. Nei's genetic distance matrix (Table 2) was calculated according to Nei, 1972.

To identification the correlations among individuals in the subpopulation used to calculated Fis, the frequency correlations among subpopulations to Fst, and for both factor was Fit. $\mathrm{Nm}$ analysis was a total of migrant and polymorphism information content with PIC. The SSR allele frequency data graphs for each locus and allelic pattern across populations are described in Figures 2 and 3. The genetic structure and population were calculated with anAMOVA to molecular variance analysis with GENALEX ver 6.502 (Peakall \& Smouse, 2006).

Statistical analyses of microsatellite data were computed using GENALEX ver 6.502, 
F-statistics (Fis, Fit, and Fst) were estimated at each locus. The probability of being greater than zero was determined by bootstrap analysis using 1,000 replicates, with a 99\% confidence interval (Peakall \& Smouse, 2006). For all statistical tests, we chose a significance level $\alpha=0.001$.

\subsection{Clustering analysis}

To identify groups of population analysis to genetic differences, we used clustering sampling by MVSP ver 3.22, Kovach computing services (UK) in unweighted pair group method of arithmetic averages (UPGMA) (Backeljau et al., 1996). The bootstrap analysis with 1,000 replication was used to assess the strength of nodes (Felsenstein, 1985).

\section{Results and discussion}

\subsection{Genetic structure analysis}

The genetic structure of the population can provide critical information for developing management strategies. PCR product analysis with SSR markers obtained an alternative method of characterization in the DNA genome. This marker was employing oligonucleotides-SSR at one or the other of the $5^{\prime}$ or $3^{\prime}$ end, with 2 to 4 purine or pyrimidine residue to start the amplification (Zietkiewicz et al., 1994). In this study, the genetic structure distribution between and within the types of Elaeis guineensis population is essential to adopt efficient strategies to screen resistance to Ganoderma.

The allele frequencies and the total number of migrant and polymorphism information content (PIC) for the four loci are given in Table 1. The locus showed disjunct distributions as the allele frequency value was not different in Fis and Fit. Genotype frequencies from loci within single populations can be represented by the fixation index, Fis $=1.00$, significant deficiency of heterozygosity (Soltis \& Soltis, 1989). In other research, the polymorphisms at 15 microsatellite loci throughout the Hevea brasiliensis genome in Southwest Amazonia were found the genetic structure, the average 21.7 alleles per locus which heterozygosity (Guen et al., 2009).

However, significant differences in Fst were observed from the locus; the values were found in 0.29 to 0.30 . Genetic differentiation among oil palm populations was decreased along a locus P4(EGIFR) and P7(EG02), indicating that locus was more similar to each other than with the locus P6(EG01) and P9(EG03). This could be explained by gene flow and balanced selective resistance. Hayati et al., 2004, reported the differences in Fst values among oil palm populations in Africa due to restricted gene flow and ecotypic selection among geographic zones. Furthermore, the observed $\mathrm{Nm}$ diversity values ranged from $0.58-0.60$. The PIC values were showed an 0.70 for locus.

Table 1. Wright's indices a Fis, Fst, Fit, Nm, and PIC.

\begin{tabular}{||cccccc||}
\hline Locus & Fis & Fst & Fit & Nm & PIC \\
P4(EGIFR) & 1.00 & $0.29 * *$ & 1.00 & 0.62 & 0.70 \\
P6(EG01) & 1.00 & $0.30 * *$ & 1.00 & 0.58 & 0.70 \\
P7(EG02) & 1.00 & $0.29 * *$ & 1.00 & 0.61 & 0.70 \\
P9(EG03) & 1.00 & $0.30 * *$ & 1.00 & 0.59 & 0.70 \\
Mean & 1.00 & 0.29 & 1.00 & 0.60 & 0.70 \\
SE & 0 & 0.04 & 0 & 0.01 & 0 \\
\hline
\end{tabular}

*Wright's statistics according to Weir and Cockerham, 1984. $\quad * * \mathrm{P}<0.001$. Fis: Correlations of alleles frequency among individuals in the population; Fst: Correlations of alleles frequency among sub-population; Fit: Correlations by both factors from alleles frequency; Nm: Total by migrant; PIC: Polymorphic information content.

The SSR marker assays were showed no significant locus differences between the PIC values. We found that the high polymorphism of genetic similarity among the seedling and mature plants of Ganoderma resistance varieties indicates that they maintain $\mathrm{PIC}=0.70$ as a result of the selection. These results are in agreement with the recent studies, and there were obtained with SSR markers. The high average $\mathrm{PIC}=0.73$ was detected polymorphism 
within the different seedling and mature oil palm sources evaluation; similarly, further confirmation of the high polymorphism of the selected SSR markers employed has been reported (Billotte et al., 2005; Singh et al., 2008).

Furthermore, the genetic relationship among date palm (Phoenix dactylifera L.) cultivars with polymorphism percentage $(36.49 \%)$ and PIC $>0.50$ by eight ISSR primers have been reported (Aladadi et al., 2018). The PIC values of E. guineensis type cold-tolerance have been reported $\mathrm{PIC}=0.65$ (Xiao et al., 2014), whereas the genetic diversity of $E$. oleifera reported PIC $=0.63$ (Zaki et al., 2012). Besides, not similarly, very low polymorphism in $E$. guineensis populations of seedling and mature species have been reported elsewhere, e.g., $\mathrm{PIC}=0.30$ in SSR marker (Babu et al., 2019). Different from the lowest PIC value, 0.16 was found in Rhanterium epapposum using SRAP marker (Al-Salameen et al., 2014).

Table 2. The pairwise population of Fst values.

\begin{tabular}{|lllllllll||}
\hline Population & SR & SN & SRIG & SNIG & MRs & MRN & MNs & MNN \\
SR & 0 & & & & & & & \\
SN & 0.19 & 0 & & & & & & \\
SRIG & 0.20 & 0.20 & 0 & & & & & \\
SNIG & 0.20 & 0.18 & 0.20 & 0 & & & & \\
MRs & 0.18 & 0.20 & 0.20 & 0.20 & 0 & & & \\
MRN & 0.20 & 0.20 & 0.20 & 0.20 & 0.15 & 0 & & \\
MNs & 0.16 & 0.20 & 0.17 & 0.20 & 0.17 & 0.18 & 0 & \\
MNN & 0.20 & 0.20 & 0.20 & 0.18 & 0.18 & 0.18 & 0.18 & 0 \\
\hline
\end{tabular}

Noted: Seedling resistance (SR), seedling non-resistance (SN), seedling resistance inoculation Ganoderma (SRIG), seedling non-resistance inoculation Ganoderma (SNIG), mature resistance symptom (MRs), mature resistance non-symptom (MRN), mature non-resistance symptom

(MNs), mature non-resistance non-symptom (MNN).

Besides, there was a significant correlation in $\mathrm{P}<0.001$ between the genetic identity and Fst across the population (Table 2). In Fst to the expected distribution of pairwise differences, the correlations caused the observed distributions to differ across the population.

For the two primers (P4 and P7), the same value was shown at the Fst $=0.29$, respectively, and the highest in P6 and P9 $(\mathrm{Fst}=0.30)$. Fst was an analysis based revealed a high genetic differentiation (Fst $>0.25$ ) among oil palm populations, respectively. However, the differences in Fst values among the total genetic differences among oil palm seedling and mature populations mainly due to restricted gene flow and resistance selection.

Loveless \& Hamrick, 1984, have reported that genetic differentiation among populations is principally a function of gene flow between populations by pollen and seeds dispersal. The species with discrete or isolated populations experiences less gene flow than species with more continuously distributed or contiguous populations and therefore have a relatively lower level of variation within populations and a higher variation among populations.

In this study, the highest levels of population differentiation were supported by low levels of gene migration among populations to the resistance of Ganoderma. Genetic diversity observed was due to genetic differentiation within the sources of parental materials like in most perennial outbreeding species, which maintain most of their variation within populations (Hamrick et al., 1991).

Such a difference has been reported by Okoye et al., 2016; the significant genetic distinction of Fst $=0.18$ indicated the presence of a genetic structure between the E. guineensis from Nigeria, which used the SSR marker. At the same time, the low Fst values indicate weak population differentiation (Cvjetković et al., 2017). 
Table 3. Pairwise population the matrix of Nei's unbiased genetic identification.

\begin{tabular}{|llllllll||}
\hline \hline Population & SR & SN & SRIG & SNIG & MRs & MRN & MNs \\
SR & 0 & & & & & & \\
SN & 0.14 & 0 & & & & & \\
SRIG & 0 & 0 & 0 & & & & \\
SNIG & 0.00 & 0.13 & 0 & 0 & & & \\
MRs & 0.13 & 0 & 0 & 0 & 0 & & \\
MRN & 0 & 0 & 0 & 0 & 0.42 & 0 & \\
MNs & 0.14 & 0 & 0.14 & 0 & 0.28 & 0.14 & 0 \\
MNN & 0 & 0 & 0 & 0.13 & 0.14 & 0.13 & 0.14 \\
\hline
\end{tabular}

Noted: Seedling resistance (SR), seedling non-resistance (SN), seedling resistance inoculation Ganoderma (SRIG), seedling non-resistance inoculation Ganoderma (SNIG), mature resistance symptom (MRs), mature resistance non-symptom (MRN), mature non-resistance symptom (MNs), mature non-resistance non-symptom (MNN).

The genetic identity across the population was pairwise was calculated using Nei's unbiased of the genetic distance. The genetic identification of E. guineensis to Ganoderma resistance populations was found from 0.13 to 0.42 (Table 3 ).

The migration rates existing among seedling and mature populations were increased. The highest genetic differentiation between oil palm populations could be mainly due to years of planting differences as the populations used in this study covered resistance genes. On the other hand, the highest genetic divergence among the populations may be due to restricted pollen and seed dispersal; likewise, insects are pollinated, such as in Africa (Hartley, 1988).

The length of the DNA samples was showed in heterozygosity from DNA genomic samples to established the expected range of 176 bp -422 bp allelic for each population per locus. Allele P7 (0.67) at mature non-resistance nonsymptom (MNN), which was present at the highest frequencies in mature and seedling populations, was not detected in any other populations assayed, which most likely indicates hybridization and introgression. The presence of rare allelic variants at a particular locus in one or two populations could be largely due to drift, to a lesser extent, to adaptive genetic variants (Bakoumé et al., 2007; Thongthawee et al., 2010).
The number of alleles described genetic variation at each locus to measure genetic variability (Arora and Bhatia, 2006). In this case, that has an impact on resistance genes differentiation within the population.

The populations with a relatively high average $\mathrm{Na}$ suggest that it has good genetic variation and could be amenable to the protection of germplasm resources. Genetic diversity is closely related to adaptive power, viability, evolutionary potential, and pathogen resistance. Finally, it also showed that this species resistant to the Ganoderma boninense has the genetic potential for breeding. Shannon's information index can indicate the level of genetic diversity; a higher value indicates greater genetic diversity (Wang et al., 2014).

For experimental validation of the data, each locus to predicted differences between size alleles, which allows the polymorphic to the variant base frequency of the allele, we describe using GENEALEX software. Allelic frequencies at these loci for each population examined, including the seedling and mature, are given in Table 4.

For experimental validation of the data, each locus to predicted differences between size alleles, which allows the polymorphic to the variant base frequency of the allele, we describe using GENEALEX software. Allelic frequencies at these loci for each population examined, including the seedling and mature, are given in Table 4. 
Table 4. Allele frequencies by populations

\begin{tabular}{|cccccccccc||}
\hline Locus & Allele & SR & SN & SRIG & SNIG & MRs & MRN & MNs & MNN \\
P4 & 176 & 0 & 0 & 0 & 0 & 0 & 0 & 0 & 0.33 \\
& 205 & 0 & $0.17 *$ & 0 & 0 & 0.33 & 0.33 & 0.33 & 0.33 \\
& 241 & 0 & 0 & 0 & 0 & 0.33 & 0 & 0 & 0 \\
& 255 & 0.33 & 0.33 & 0 & 0 & 0 & 0 & 0 & 0 \\
& 272 & $0.17 *$ & 0 & $0.17^{*}$ & 0 & 0.33 & 0 & 0 & 0 \\
& 292 & 0 & 0 & $0.17^{*}$ & 0.33 & 0 & 0 & 0 & 0 \\
& 300 & 0 & 0.33 & 0 & 0 & 1.00 & 0 & 0 & 0 \\
P6 & 265 & 0 & 0 & 0 & 0.33 & 0 & 0 & 0 & 0 \\
& 277 & 0 & 0 & 0.33 & 0 & 0 & 0 & 0 & 0 \\
& 283 & 0.25 & 0 & 0 & 0 & 0 & 0 & 0 & 0.33 \\
& 294 & 0 & 0 & 0 & 0 & 0 & 0 & 0.33 & 0 \\
& 297 & 0 & 0.33 & 0 & 0 & $0.17 *$ & 0 & 0 & 0 \\
& 303 & 0.25 & 0 & 0 & 0 & $0.17 *$ & 0 & 0.50 & 0 \\
& 317 & 0 & 0 & 0 & 0 & 0 & 0.33 & 0 & 0 \\
P7 & 361 & 0 & 0 & 0 & 0 & 0.33 & 0 & 0 & 0 \\
& 293 & 0.25 & 0 & 0 & 0 & 0 & 0 & 0.33 & 0 \\
& 300 & 0.33 & 0 & 0 & 0 & 0.33 & 0 & 0.33 & 0 \\
& 316 & 0.33 & 0 & 0 & 0 & 0 & 0 & 0 & 0 \\
& 332 & 0 & 0 & 0 & 0 & 0.33 & 0.33 & 0 & 0 \\
& 347 & 0 & 0.33 & 0 & 0 & 0 & 0 & 0 & $0.67 *$ \\
& 365 & 0 & 0 & 0 & 0 & 0 & 0.33 & 1.00 & 0 \\
P9 & 203 & 0 & 0.25 & 1.00 & 0.50 & 0 & 0 & 0 & 0.33 \\
& 253 & 0.25 & 0 & 0 & 0 & 0 & 0 & 0.33 & 0 \\
& 272 & 0 & 0 & 0 & 0 & 0.50 & 0 & 0 & 0.33 \\
& 297 & 0 & 0 & 0.33 & 0 & 0 & 0.33 & 0 & 0 \\
& 315 & 0 & 0.33 & 0 & 0.33 & 0 & 0.33 & 0.50 & $0.17 *$ \\
& 326 & 0 & 0 & 0.33 & 0 & 0 & 0.33 & 0 & 0 \\
& 340 & 0.25 & 0 & 0 & 0.33 & 0 & 0 & 0 & $0.17 *$ \\
& 422 & 0 & 0 & 0 & 0 & 0.33 & 0 & 0 & 0.33 \\
\hline \hline
\end{tabular}

Noted: P4=Primer EGIFR; P6=Primer EG01; P7=Primer EG02; P9=Primer EG03. Seedling resistance (SR), seedling non-resistance (SN), seedling resistance inoculation Ganoderma (SRIG), seedling non-resistance inoculation Ganoderma (SNIG), mature resistance symptom (MRs), mature resistance non-symptom (MRN), mature non-resistance symptom (MNs), mature non-resistance non-symptom (MNN). *Significant at $\mathrm{P}$-value $<0.001$.

discriminatory power of the SSR primer combinations. Microsatellite analysis was observed between oil palm (E. guineensis) from China and Malaysia; the Ho found 0.37 and 0.40 (Zhou, 2015). While there found two to nine different Ho, an average of $0.08-0.83$ per locus in S. sclerotiorum species were polymorphic (Mahalingam et al., 2020). The variation was due to the differences in the number of microsatellite repeat motifs in each locus. In Figure 1, we were indicated by a graph of seven polymorphic loci parameter edges. 

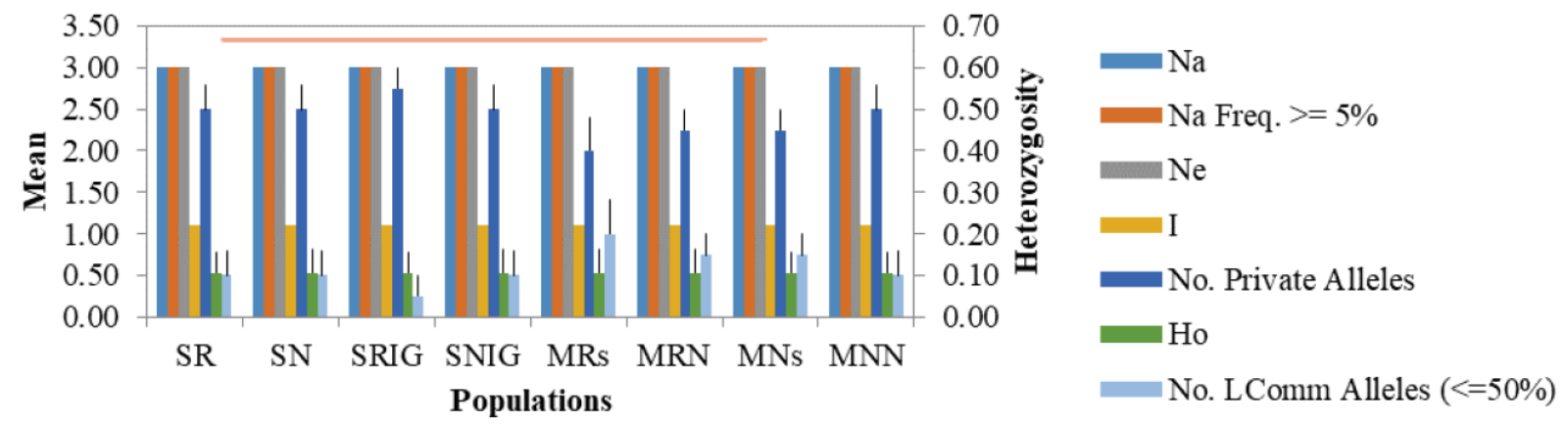

Fig. 1. Average allelic patterns across populations. (Seedling resistance (SR), seedling non-resistance (SN), seedling resistance inoculation Ganoderma (SRIG), seedling non-resistance inoculation Ganoderma (SNIG), mature resistance symptom (MRs), mature resistance non-symptom (MRN), mature non-resistance symptom (MNs), mature non-resistance non-symptom (MNN)).

Table 5. The molecular variance analysis

\begin{tabular}{||ccccccc||}
\hline \hline Source & SS & df & MS & Variance estimates & Percentage & P-Value \\
Among Pops & 26.8 & 7 & 3.8 & 0 & 0 & \\
Among Individual & 64.0 & 16 & 4.0 & 2.0 & 100 & $<0.001$ \\
Within Individual & 0 & 24 & 0 & 0 & 0 & \\
Total & 90.6 & 47 & & 2.0 & 100 & \\
\hline \hline
\end{tabular}

Noted: P-value to the level of significance for the distribution of variation.

The $\mathrm{Na}$ values for all populations are the same at 3.00. Additionally, we identify the known number of active alleles $\mathrm{Ne}=3.00 . \mathrm{v} \quad$ We were shown the graph's more certain maximum likelihood by calculating the average molecular weight in locus with an across the random population. That graph model was clarified almost $99.9 \%$ of the variance in the relative population. Furthermore, as intended from an
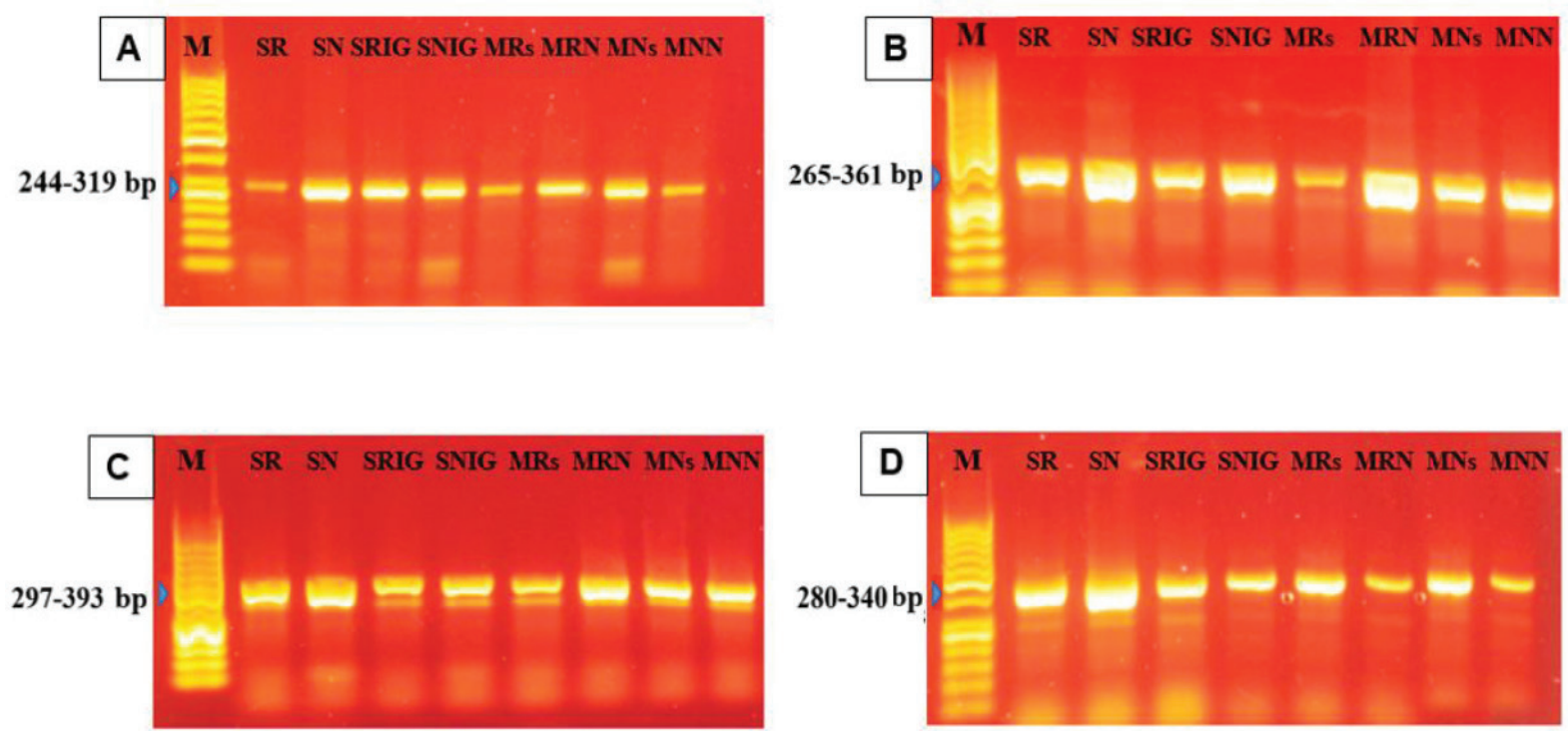

investigation of the resistance genes, it was shown that seedling and mature populations also have the resistance gene of Ganoderma To determine the genetic differences among the eight groups, we performed AMOVA and pairwise analyses (Table 5). The AMOVA results showed that differences among individuals caused $100 \%$ of the variation; it was significantly different from each other in

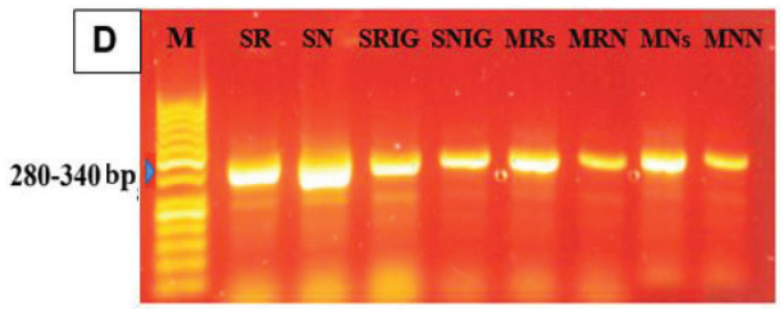

Fig. 2. Agarose gel of PCR visualization seedling populations by DNA ladder staining from $50 \mathrm{bp}$ $\sim 1000 \mathrm{bp} ; 1 \%$ agarose gel. (A=Primer P4(EGIFR), $\mathrm{B}=$ Primer P6(EG01), $\mathrm{C}=$ Primer P7(EG02) and $\mathrm{D}=$ Primer $\mathrm{P}$ 9(EG03); Seedling resistance (SR), seedling non-resistance (SN), seedling resistance inoculation Ganoderma (SRIG), seedling non-resistance inoculation Ganoderma (SNIG), mature resistance symptom (MRs), mature resistance non-symptom (MRN), mature non-resistance symptom (MNs), mature non-resistance non-symptom (MNN)). 
the seedling and mature oil palm genotype. This suggests that genetic differences between individuals can explain existing genetic diversity.

\subsection{PCR amplification}

The SSR markers are a powerful tool for this type of study. We were showed the possibility of using SSR primers to amplify in different types of seedling and mature. The putative loci were incorporated in the PCR as they can provide the resistance gene of Ganoderma recommended in the analysis to E. guineensis species (Tan et al., 2013). Moreover, another primer (P6(EG01); P7(EG02); P9(EG03)) was designed to extremely amplify the level of band expression. Four primers tested can amplify eight types of seedling and mature species by four primers' results among the total of 64 bands, whereas the seedling type was 32 bands and the mature types 32 bands.

Figures 2(A) and 2(B) were showed the amplificationappearance delivered over eight seedling populations using primer P4(EGIFR) and P6(EG01), respectively. The single bands of varied size, from $244-319 \mathrm{bp}$, were found based on primer $\mathrm{P} 4$, while, in the primer $\mathrm{P} 6$, the sizes varied from 265 to $261 \mathrm{bp}$. Likewise, a
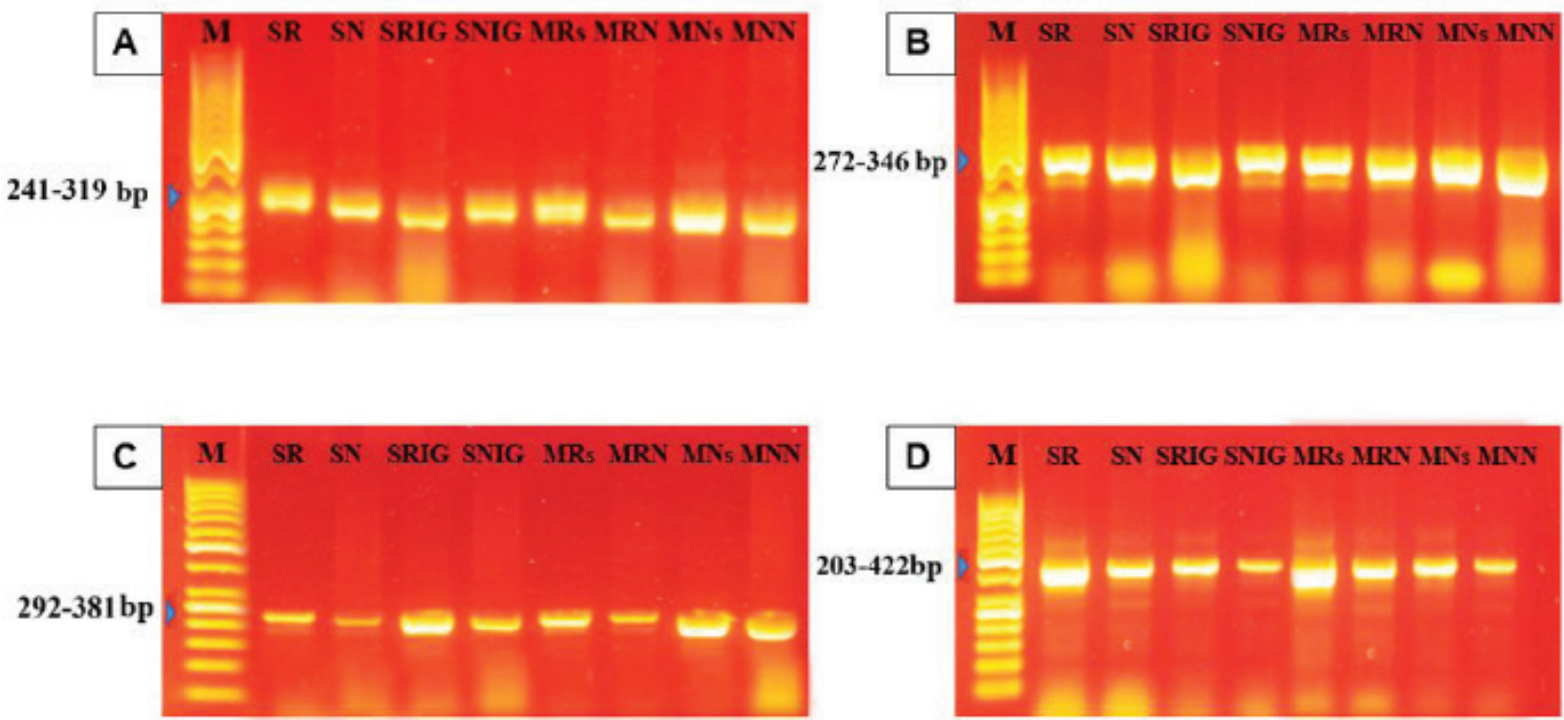

Fig. 3. Agarose gel of $\mathrm{PCR}$ visualization mature populations by DNA ladder staining from $50 \mathrm{bp}$ 1000 bp; 1\% agarose gel. (A=Primer P4(EGIFR), B=Primer P6(EG01), $\mathrm{C}=$ Primer P7(EG02) and $\mathrm{D}=$ Primer P9(EG03); Seedling resistance (SR), seedling non-resistance (SN), seedling resistance inoculation Ganoderma (SRIG), seedling non-resistance inoculation Ganoderma (SNIG), mature resistance symptom (MRs), mature resistance non-symptom (MRN), mature non-resistance symptom (MNs), mature non-resistance non-symptom (MNN)). 


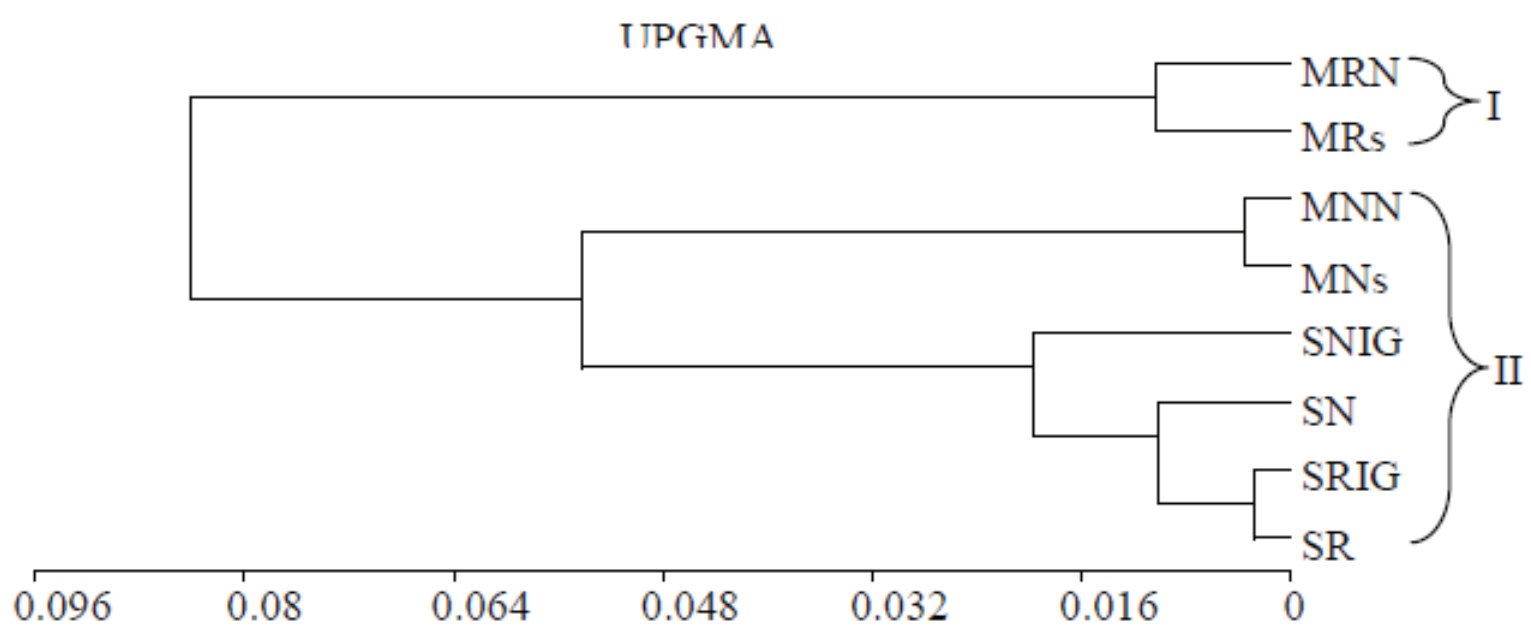

Fig. 4. Dendrogram based on Squared Euclidean - Datalog(10) transformed genetic distances using the pairwise unweighted clustering method (UPGMA, MVSP ver 3.22). Seedling resistance (SR), seedling non-resistance (SN), seedling resistance inoculation Ganoderma (SRIG), seedling non-resistance inoculation Ganoderma (SNIG), mature resistance symptom (MRs), mature resistance non-symptom

(MRN), mature non-resistance symptom (MNs), mature non-resistance non-symptom (MNN).

amplification data from the marker by SSR was used to estimate the genetic distance and to draw a phylogenetic tree. The dendrogram UPGMA algorithm was used to clustering the population based on the genetic distances population.

The bootstrapping value represents a percentage in 1,000 to replications and verifies the reliability of the topology tree, mainly in a manner appropriate to SSR markers. The phylogenetic tree constructed from the SSR markers data was displayed two (2) clustering. The first cluster includes the mature resistance non-symptom (MRN) and the mature resistance symptom (MRs) were separated. Furthermore, in the second cluster, the lines from seedling E. guineensis were derived, i.e., seedling resistance (SR), seedling non-resistance (SN), seedling resistance inoculation Ganoderma (SRIG), and seedling non-resistance inoculation Ganoderma (SNIG), all of which exhibited a close relationship in one group. Furthermore, the mature non-resistance symptom (MNs) and mature non-resistance non-symptom (MNN) displayed to the out-group separately.

The population in this study was not divided into separate clusters, but it was distributed in two large clusters, which showed a significant genetic similarity of the population. It was seen that the seedling population was more closely related; the seedling resistance inoculation and the non-inoculation were separated into one large group. Cluster analysis has separated the seedling and matures to Ganoderma resistance populations also different with respect to the genetic background of MTG variety traits in that they have a tolerance gene for Ganoderma (Putri et al., 2017). Based on these unique characteristics, there should be attempts to classify the oil palm as a different species (Purseglove, 1975; Hartley, 1988).

Microsatellite analysis efficiently was differentiated oil palm type seedlings and matured to Ganoderma resistance. These results will be useful for further studies, such as material planting selection, clonal identification, the monitoring of progeny in seed-production programs, and genetic mapping. Hama-Ali et al., 2014, reported that microsatellite analysis has successfully revealed the polymorphism associated with seedling and mature variation in the oil palm resistance gene to Ganoderma. Nevertheless, the seedling and mature variants were identified as their biochemistry characteristics (Afandi et al., 2018), but they have been developed were clarification by this research.

Finally, oil palm can be distributed mainly in the tropical area of North Sumatra. Hence, 
Sumatera oil palm's development history and the experimental findings support the conclusion that the populations of Ganoderma resistance may be from the same source, and they have a similar genetic background. Further research was needed in this area. The genetic diversity and population structure data based on microsatellite analysis of SSR were a theoretical basis for deeper study and research into oil palm breeding. In the process of breeding selection, other reference indices, such as fruit productivity, disease control, and pathogen stress, should be used to determine breeding materials in the same group. Microsatellite molecular systematic data can contribute to the development of effective Ganoderma strategies.

The genetic data was obtained here for the oil palm-based microsatellite markers demonstrated the genetic structure indirectly. These data were provided the genetic information for eight population seedlings and matured based on resistance Ganoderma oil palm lines that a great amount of genetic structure variation in the oil palm to preserved and improvement of oil palm breeding.

\section{Conclusion}

This study was confirmed the seedling and maturation of Elaeis guineensis Jacq. using microsatellite analysis. Four primers were amplified the PCR product of seedling and mature species, the single bands of varied sizes, a substantial range of the variability caused by small implied deletion from the genomic regions amplified among the microsatellite anchored in the regions. This study was confirmed the high polymorphism of genetic similarity among the seedling and mature of oil palm plants to Ganoderma resistance.

The genetic variation among the eight populations caused by differences among individuals was significantly different from each other. The separate phylogenetic clusters were distributed in two large clusters, which showed a significant genetic similarity of the population. The seedling population was found more closely related; the seedling resistance inoculation and the noninoculation were separate in one large group.

\section{ACKNOWLEDGEMENTS}

This study was funded by the Directorate of Research and Technology and Higher Education, (No.152/SP2H/LT/DPRM/2019) through PMDSU (Pendidikan Magister Menuju Doktor Untuk Sarjana Unggul) Program, Republic of Indonesia.

\section{References}

Afandi D.; Basyuni, M.; Putri, L.A.P.; Chalil, D. \& Syahputra, I. (2018) Expression of oil palm (Elaeis guineensis) polyisoprenoids in response to Ganoderma boninense infection. Biodiversitas, 20(1): 68-76.

Aladadi, W.M.; Mahmoud, F.; Moustafa, Sulaiman, A.; Alruman. (2018) Genetic variability among seven cultivars of date palm (Phoenix dactylifera L.) based on embryonic DNA of old fruit. Kuwait Journal of Science, 45 (1): 108-114.

Al-Salameen, F.; Habibi, N.; Kumar, V.; Al-Amad, S.; Dashti, J. et al. (2018) Genetic diversity and population structure of Haloxylon salicornicum moq. in Kuwait by ISSR markers. PloS one, 13(11): 69.

Arora, R. \& Bhatia, S. (2006) Genetic diversity of Magra sheep from India using microsatellite analysis. Journal of Animal Science, 19: 938-942.

Babu, K.B.; Mary, R.K.L.; Sahu, S.; Mathur, R.K.; Kumar, P.N. et al. (2019) Development and validation of whole genome-wide and genic microsatellite markers in oil palm (Elaeis guineensis Jacq.): First microsatellite database (OpSatdb). Scientific Reports, 9(1): 1-9. 
Backeljau, T.; Bruyn, D.L.; Wolf, D.H.; Jordaens, K.; Dongen, V. et al. (1996) Multiple UPGMA and neighbor-joining trees and the performance of some computer packages. Molecular Biology and Evolution, 13(2): 309-313.

Bakoumé, C.; Wickneswari, R.; Rajanaidu, N.; Kushairi, A.; Amblard, P. et al. (2007) Allelic diversity of natural oil palm (Elaeis guineensis Jacq.) populations detected by microsatellite markers: implications for conservation. Plant Genetic Resources, 5(2): 104-107.

Bakoumé, C.; Wickneswari, R.; Siju, S.; Rajanaidu, N.; Kushairi, A. et al. (2015) Genetic diversity of the World's largest oil palm (Elaeis guineensis Jacq.) field Genebank Accessions using microsatellite markers. Genetic Resources and Crop Evolution, 62: 349- 360.

Barcelos, E.; Rios, S.D.A.; Cunha, R.N.; Lopes, R.; Motoike, S.Y. et al. (2015) Oil palm natural diversity and the potential for yield improvement. Frontiers in Plant Science, 6: 190 .

Billotte, N.; Marseillac, N.; Risterucci, A.; Adon, B.; Brottier, P. et al. (2005) Microsatellite-based high-density linkage map in oil palm (Elaeis guineensis Jacq.). Theoretical and Applied Genetics, 110: 754765.

Caro, M.L.; Rimbawanto, A., \& Page, D.E. (2014) Management of basidiomycete root and stem rot diseases in oil palm, rubber, and tropical hardwood plantation crops. Pathology, 44: 428-446.

Cochard, B.; Adon, B.; Rekima, S.; Billotte, N.; Desmier, R. et al. (2009) Geographic and genetic structure of African oil palm diversity suggests new approaches to the breeding tree. Genetics and Genomes, 5: 493-504.
Cvjetković, B.; Konnert, M.; Fussi, B.; Mataruga, M.; Nikolić, S.M. et al. (2017) Norway spruce (Picea abies Karst.) variability in progeny tests in Bosnia and Herzegovina. Genetika, 49(1): 259-272.

Erlich, A.; Gelfand, D. \& Sninsky, J.J. (1991) Recent advances in the polymerase chain reaction. Recent, (7): 1643-1652.

Faizah, R.; Putranto, R. A.; Wening, S.; Sukma, D.; Raharti, V.R. et al. (2020) Differential expression of root-specific genes of oil palm seedlings at an early stage of Ganoderma boninense infection. IOP Conference Series: Earth and Environmental Science, 418: 44.

Felsenstein, J. (1985) Confidence limits on phylogenies: an approach using the bootstrap. Evolution, 39: 783-791.

Guen, L.V.; Doaré, F.; Weber, C., \& Seguin, M. (2009) Genetic structure of Amazonian populations of Hevea brasiliensis is shaped by hydrographical network and isolation by distance. Tree genetics \& genomes, 5(4): 673-683.

Hama-Ali, E.O.; Panandam, J.M.; Tan, S.G.; Alwee, S.S.R.S.; Sheong, T.J. et al. (2014) Association between basal stem rot disease and simple sequence repeat markers in oil palm, Elaeis guineensis Jacq. Euphytica, 202(2): 199-206.

Hamrick, J.L. \& Murawski, D.A. (1991) Levels of allozyme diversity in populations of uncommon neotropical tree species. Journal of Tropical Ecology, 7: 395-399.

Hartley, C.W.S. (1988) The oil palm (Elaeis guineensis Jacq.). Longman Scientific and Technical Publ. New York.

Hayati, A.; Wickneswari, R.; Maizura, I., \& Rajanaidu, N. (2004) Genetic diversity of oil palm (Elaeis guineensis Jacq.) germplasm 
collections from Africa: implications for improvement and conservation of genetic resources. Theoretical and Applied Genetics, 108(7): 1274-1284.

Hayati, R.; Basyuni, M., \& Chalil, D. (2020) Genetic diversity, sequence and bioinformatic analysis of Ganoderma boninense isolates. International Journal of Agriculture and Biology, 23(4): 763-770.

Indonesian Directorate General of Estate. (2020) Palm oil production by province in Indonesia. Directorate General of Estate: 214.

Lee, P.Y.; Costumbrado, J.; Hsu, C.Y. \& Kim, Y.H. (2012) Agarose gel electrophoresis for the separation of DNA fragments. Journal of Visualized Experiments, 62: 1-5.

Loveless, M.D. \& Hamrick, J.L. (1984) Ecological determinants of genetic structure in plant populations. The Annual Review of Ecology, Evolution, and Systematics, 15: 65-95.

Madar, U.; Thangadurai, D.; Kulkarni, S.S.; Gai, P. \& Saliyavar, B. (2019) Genetıc varıation in arenga wightı griff . (Arecaceae) populations using inter simple sequence repeat (ISSR) markers. Plant Archives, 19(1): 1646-1652.

Mahalingam, T.; Chen, W.; Rajapakse, C.S.; Somachandra, K.P., \& Attanayake, R.N. (2020) Genetic Diversity and Recombination in the Plant Pathogen Sclerotinia sclerotiorum Detected in Sri Lanka. Pathogens, 9(4): 306.

Maughan, P.J.; Maroof, M.A. \& Buss, G.R. (1995) Microsatellite and amplified sequence length polymorphisms in cultivated and wild soybean. Genome, 38(4): 715-723.

Mudge, A.; Rama, D.; Pilotti C. \& Godwin, I. (2020) Genetic diversity and population structure of field isolates of Ganoderma boninense from oil palm plantation in the Solomon Islands. Proceedings, 36(1): 56.
Mullis, K.B. (1990) The unusual origin of the polymerase chain reaction. Scientific American, 262(4): 56-65.

Nei, M. (1972) Genetic distance between populations. The American Naturalist, 106(949): 283-292.

Neuhauser, S.; Huber, L. \& Kirchmair, M. (2009) A DNA-based method to detect the grapevine root-rotting fungus Roesleria subterranean in soil and root samples. Phytopathologia Mediterranea, 48(1): 5972.

Okoye, M.N.; Bakoumé, C.; Uguru, M.I.; Singh, R. \& Okwuagwu, C.O. (2016) Genetic relationships between elite oil palms from Nigeria and selected breeding and germplasm materials from Malaysia via simple sequence repeat (SSR) Markers. Journal of Agricultural Science, 8(2): 159.

Osorio-Guarín, J.A.; Garzón-Martínez, G.A.; Delgadillo-Duran, P.; Bastidas, S.; Moreno, L.P. et al. (2019) Genomewide association study (GWAS) for morphological and yield-related traits in an oil palm hybrid (Elaeis oleifera $\times$ Elaeis guineensis) population. BMC Plant Biology, 19(1): 1-11.

Peakall, R. and Smouse, P.E. (2006) GENALEX 6: Genetic analysis in Excel. Population genetic software for teaching and research. Molecular Ecology Notes, 6(1): 288-295.

Phalan, B.; Onial, M.; Balmford, A., and Green, R.E. (2011) Reconciling food production and biodiversity conservation: land sharing and land sparing compared. Science, 333: 1289-1291.

Powell, W.; Morgante, M.; Andre, C.; Hanafey, M.; Vogel, J. et al. (1996) The comparison of RFLP, RAPD, AFLP and SSR (microsatellite) markers for germplasm analysis. Molecular Breeding, 2: 225-238. 
Purba, A.; Basyuni, M.; Putri, L.A.; Chalil, D.; Hayati, R. et al. (2019) Sequence analysis of Ganoderma boninense isolates from oil palm. IOP Conference Series: Earth and Environmental Science, 260: 012172.

Purseglove, J.W. (1975) Tropical crops, monocotyledons. The English Language Book Society and Longman Scientific Publ, London.

Putri, L.A.P.; Setiado, H., \& Hardianti, R. (2017) DNA Profiles of MTG (Moderat Tahan Gano) oil palm variety based on SSR marker. IOP Conference Series: Materials Science and Engineering 180(1): p. 012044.

Rana, N. \& Jain, S. (2019) DNA quantification of wild and cultured Labeo Rohita (Hamilton, 1822) using nanophotometer. Journal of Experimental Zoology of India, 22(1): 453-455.

Saktioto, T.; Suhardi, S.; Defrianto, D.; Roslim, D.I., \& Awitdrus, A. (2019) Stimulating ion flow in oil palm leaves and midribs applying electrical potential difference. Kuwait Journal of Science, 46: 1.

Sayer, J.J.; Ghazoul, Nelson P.; Boedhihartono, A.K. (2012) Oil palm expansion transforms tropical landscapes and livelihoods. Global Food SecurityAgriculture Policy Economics and Environment, 1: 114-119.

Shahbandeh, M. (2020) Production volume of palm oil worldwide from 2012/2013 to 2019/2020. Statista: 613471.

Singh, R.; Noorhariza, M.Z.; Ting, N.C.; Rozana, R.; Tan, S.G. et al. (2008) Exploiting an Oil Palm EST Database for the Development of Gene-derived SSR Markers and their exploitation for Assessment of Genetic Diversity. Biologia, 63:1-9.
Soltis, D.E. \& Soltis, P.S. (1989) Polyploidy, breeding systems and genetic differentiation in homosporous pteridophytes. In: Soltis DE, Soltis PS (eds) Isozymes in plant biology. Dioscorides Press, Portland, Ore., 1: 241258.

Sserumaga,J.P.; Makumbi,D.; Warburton, M.L.; Opiyo, S.O.; Asea, G. et al. (2019) Genetic diversity among tropical provitamin A maize inbred lines and implications for a biofortification program. Cereal Research Communications, 47(1): 134-144.

Tan, Y.C.; Yeoh, K.A.; Wong, M.Y. \& Ho, C.L. (2013) Expression profiles of putative defense-related proteins in oil palm (Elaeis guineensis) colonized by Ganoderma boninense. Journal of Plant Physiology, 170(16): 1455-1460.

Teh, C.K.; Sudirman, N.A.; Rodzik, F.F.M.; Ong, A.L.; Kwong, Q.B. et al. (2020) Genetic Dissecting Complex Traits via Conventional QTL Analysis and Association Mapping. Springer, Cham. The Oil Palm Genome 1: 131-140.

Terryana, R.T.; Nugroho, K. \& Lestari, P. (2020) Genetic diversity of sugar palm populations from Cianjur and Banten revealed by simple sequence repeat (SSR) Markers. IOP Conference Series: Earth and Environmental Science, 418: 012038.

Thongthawee, S.; Tittinutchanon, P., \& Volkaert, H. (2010) Microsatellites for parentage analysis in an oil palm breeding population. Genomics and Genetics, 3(2): 172-181.

Voora, V., Larrea, C., Bermudez, S., \& Baliño, S. (2020) Global Market Report: Palm Oil. International Institute for Sustainable Development, Winnip eg, Canada. 
Wang, S.; Liu, Y.; Ma, L.; Liu, H. (2014) Isolation and characterization of microsatellite markers and analysis of genetic diversity in Chinese jujube (Ziziphus jujuba Mill.). PLoS One, 9: e99842.

Weir, B. S.; Cockerham, C.C. (1984) Estimating F-statistics for the analysis of population structure. Evolution, 38: 13581370.

Xiao, Y.; Zhou, L.; Xia, W.; Mason, A.S.; Yang, Y. et al. (2014) Exploiting transcriptome data for the development and characterization of gene-based SSR markers related to cold tolerance in oil palm (Elaeis guineensis). BMC Plant Biology, 14(1): $1-13$.

Zaki, N.M.; Singh, R.; Rosli, R. \& Ismail, I. (2012) Elaeis oleifera genomicSSR markers: Exploitation in oil palm germplasm diversity and cross-amplification in Arecaceae. International Journal of Molecular Sciences, 13(4): 4069-4088.

Zeugin, J.A. \& Hartley, J.L. (1985) Ethanol precipitation of DNA. Biophysics, 42: 105-110.

Zhou, L.X.; Xiao, Y.; Xia, W., \& Yang, Y.D. (2015) Analysis of genetic diversity and population structure of oil palm (Elaeis guineensis) from China and Malaysia based on species-specific simple sequence repeat markers. Genetic and Molecular Research, 14(4): 16247-16254.

Zietkiewicz, E.; Rafalski, A. \& Labuda, D. (1994) Genome fingerprinting by simple sequence repeat (SSR)-anchored polymerase chain reaction amplification. Genomics, 20: 176-183.

Submission:

DOI: $10.48129 /$ kjs.v48i3.9382 